\title{
BMJ Open Point-of-care ultrasonography for risk stratification of non-critical COVID-19 patients on admission (POCUSCO): a study protocol of an international study
}

\author{
François Morin (DD , ${ }^{1}$ Delphine Douillet, ${ }^{1,2}$ Jean-Francois Hamel, ${ }^{3}$ \\ Josué Rakotonjanahary, ${ }^{4}$ Florence Dupriez, ${ }^{5}$ Dominique Savary, ${ }^{1,6}$ \\ Christophe Aubé, ${ }^{7}$ Jeremie Riou, ${ }^{3}$ Vincent Dubée (D) , ${ }^{8}$ Pierre-Marie Roy (1) ${ }^{1,2}$
}

To cite: Morin F, Douillet D, Hamel J-F, et al. Point-ofcare ultrasonography for risk stratification of noncritical COVID-19 patients on admission (POCUSCO): a study protocol of an international study. BMJ Open 2021;11:e041118. doi:10.1136/ bmjopen-2020-041118

- Prepublication history for this paper is available online. To view these files, please visit the journal online (http://dx.doi. org/10.1136/bmjopen-2020041118).

Received 03 June 2020 Revised 05 January 2021 Accepted 21 January 2021

Check for updates

(C) Author(s) (or their employer(s)) 2021. Re-use permitted under CC BY-NC. No commercial re-use. See rights and permissions. Published by BMJ.

For numbered affiliations see end of article.

Correspondence to

Dr François Morin;

Francois.Morin@chu-angers.fr

\section{ABSTRACT}

Introduction In the context of the COVID-19 pandemic, early identification of patients who are likely to get worse is a major concern. Severity mainly depends on the development of acute respiratory distress syndrome (ARDS) with a predominance of subpleural lesions. Lung point-of-care ultrasonography (L-POCUS) is highly effective in detecting pulmonary peripheral patterns and may be appropriate for examining patients with COVID-19. We suggest that L-POCUS performed during the initial examination may identify patients with COVID-19 who are at a high risk of complicated treatment or unfavourable evolution.

Methods and analysis Point-of-care ultrasonography for risk stratification of non-critical COVID-19 patients on admission is a prospective, multicentre study. Adult patients visiting the emergency department (ED) of participating centres for suspected or confirmed COVID-19 are assessed for inclusion. Included patients have LPOCUS performed within 48 hours following ED admission. The severity of lung damage is assessed using the LPOCUS score based on 36 points for ARDS. Apart from the L-POCUS score assessment, patients are treated as recommended by the WHO. For hospitalised patients, a second L-POCUS is performed at day $5 \pm 3$. A follow-up is carried out on day 14 , and the patient's status according to the Ordinal Scale for Clinical Improvement for COVID-19 from the WHO is recorded.

The primary outcome is the rate of patients requiring intubation or who are dead from any cause during the 14 days following inclusion. We will determine the area under the ROC curve of L-POCUS.

Ethics and dissemination The protocol has been approved by the French and Belgian Ethics Committees and is carried out in accordance with the Declaration of Helsinki and Good Clinical Practice guidelines. The study is funding by a grant from the French Health Ministry, and its findings will be disseminated in peer-reviewed journals and at scientific conferences.

Trial registration number NCT04338100.

\section{BACKGROUND}

The COVID-19 pandemic has developed worldwide in less than 4 months. ${ }^{12}$ While
Strengths and limitations of this study

- Lung point-of-care ultrasonography (L-POCUS) is a simple and non-invasive tool, currently used in everyday clinical practice, that may be an alternative to CT scan as a prognostic tool in patients with suspected or confirmed COVID-19.

- Point-of-care ultrasonography for risk stratification of non-critical COVID-19 patients on admission (POCUSCO) is a prospective cohort study aimed to assess the value of L-POCUS to identify patients who are at a high risk of adverse clinical outcomes.

- The study will focus on the initial exam of patients with suspected or confirmed mild to moderate COVID-19.

- The primary outcome, a composite of death or intubation within 14 days after inclusion, is clinical and consensual.

- POCUSCO is, in our knowledge, the first prospective study on this specific topic, but its results should be confirmed in a formal implementation trial.

most patients have a mild or uncomplicated form of the disease $(80 \%)$, approximately $15 \%$ need hospital care and $5 \%$ intensive care. $^{3}$ Severe cases are characterised by pulmonary involvement that may progress to acute respiratory distress syndrome (ARDS), usually between day 7 and day $10 .{ }^{4}$ Early identification of patients who are likely to get worse is therefore a major concern.

While chest X-ray has poor diagnostic performances, ${ }^{4}$ pulmonary CT (CT scan) appears to be very sensitive $(97 \%)$ and quite specific to COVID-19 in patients with clinical suspicion of COVID-19, provided that it is not performed within the first 4 days after symptom onset. $^{56}$ A subpleural bilateral ground-glass pattern can precede the positivity of RT-PCR for SARS-CoV-2. ${ }^{7}$ In retrospective studies, quantitative CT scan analysis, using a CT scoring method, seems to accurately assess 
the severity and predict mortality of COVID-19 patients. ${ }^{89}$ Therefore, CT scan is now considered as the best imaging test to assess COVID-19 patients and is recommended as a first-line diagnostic tool by national societies of radiology ${ }^{10-12}$ However, performing CT scans for all or many patients with suspected COVID-19 may result in radiology departments being overwhelmed, especially considering bio-cleaning between patients. Moreover, CT scans may lead to adverse effects including induced cancer due to the cumulative diagnostic irradiation.

Chest ultrasonography may be an alternative to CT scans as a prognosis tool. It is simple, non-invasive, nonirradiating, inexpensive and available at the point-of-care ultrasound (POCUS). Most emergency physicians and many other specialists (pneumologists, infectious disease and intensive care physicians) are trained to perform lung-POCUS (L-POCUS) and use it in their everyday practice. Multiple studies have demonstrated its superiority to chest X-ray in detecting pneumonia. ${ }^{13}$ In ARDS, a scoring system has been developed and has shown good correlation with mortality. ${ }^{1415}$

L-POCUS is highly effective in detecting peripheral patterns and pleural abnormalities and seems appropriate to examine patients with COVID-19. ${ }^{16} \mathrm{~A}$ recent review confirms that most of patients with COVID-19 have L-POCUS abnormalities in correlation with CT findings and highlights its potential value in help-decision making for triage or follow-up. ${ }^{17}$ However, the performances of L-POCUS to predict an unfavourable outcome are still unclear and remain to be confirmed in a large prospective study.

\section{Aims and hypothesis}

Our main hypothesis is that L-POCUS performed during the initial examination may identify high-risk patients with COVID-19 and lead to close monitoring of those patients. The key secondary aim is to evaluate the risk of unfavourable outcome over time and whether L-POCUS performances vary depending on time. The other secondary aim is to determine risk stratification threshold values and classify three levels of risk: low-risk, intermediate-risk and high-risk patients. The last secondary aim is to evaluate if adding value of L-POCUS score to previous risk stratification clinical rules (qSOFA, CRB65 and CURB 65) that have been developed in order to predict death of adult patients with COVID-19, ${ }^{18}$ making it possible to identify more precisely high-risk patients.

\section{METHODS/DESIGN}

\section{Study design}

Point-of-care ultrasonography for risk stratification of COVID-19 patients (POCUSCO) is a non-interventional, prospective, multicentre study conducted by Angers University Hospital (France) and led in 11 participating centres across France and Belgium. The study was registered with ClinicalTrials.gov on 4 April 2020.
Table 1 Organisationscale of clinical improvement of the WHO

\begin{tabular}{|c|c|c|}
\hline Patient state & Descriptor & Score \\
\hline Uninfected & $\begin{array}{l}\text { No clinical or virological evidence } \\
\text { of infection. }\end{array}$ & 0 \\
\hline \multirow[t]{2}{*}{ Ambulatory } & No limitation of activities. & 1 \\
\hline & Limitation of activities. & 2 \\
\hline \multirow[t]{2}{*}{$\begin{array}{l}\text { Hospitalised } \\
\text { mild disease }\end{array}$} & $\begin{array}{l}\text { Hospitalised and no oxygen } \\
\text { therapy. }\end{array}$ & 3 \\
\hline & Oxygen by mask or nasal prongs. & 4 \\
\hline \multirow[t]{3}{*}{$\begin{array}{l}\text { Hospitalised } \\
\text { severe disease }\end{array}$} & $\begin{array}{l}\text { Non-invasive ventilation or high- } \\
\text { flow oxygen. }\end{array}$ & 5 \\
\hline & $\begin{array}{l}\text { Intubation and mechanical } \\
\text { ventilation. }\end{array}$ & 6 \\
\hline & $\begin{array}{l}\text { Ventilation+additional organ } \\
\text { support: pressors, renal } \\
\text { replacement therapy and ECMO. }\end{array}$ & 7 \\
\hline Dead & Death. & 8 \\
\hline
\end{tabular}

ECMO, Extracorporeal Membrane Oxygenation; WHO, World Health Organization.

\section{Study settings and population}

Participation in the study is proposed to patients referred to or hospitalised in 1 of the 11 participating centres from France and Belgium. Patients are screened, and if the patients fulfil all inclusion criteria and none of the study's non-inclusion criteria, written information is given, and non-opposition consent is collected. A systematic lung ultrasonography exam is performed on every study patient, and an L-POCUS score ranging from 0 to 36 points is given. Apart from the L-POCUS score assessment, patients are treated as usual according to local procedures in participating hospitals. ${ }^{19}$ Patient then either returns home or is hospitalised. For hospitalised patients, if possible, a second chest ultrasonography is performed on day $5 \pm 3$ days. The extent of lung damage is assessed by the L-POCUS score. A follow-up is carried out on day 14, and the patient's status according to the Ordinal Scale for Clinical Improvement for COVID-19 from the WHO is recorded (table 1). ${ }^{20}$

\section{Inclusion criteria}

For this study, adult patients ( $\geq 18$ years old) with COVID-19 that is confirmed by positive SARS-CoV-2 RT-PCR, suggested by typical CT scan lesions or considered as probable by the in-charge physician, are recruited. Patients should not require respiratory assistance and/or other intensive care and should not be subject to a limitation of treatments. Patients must also be beneficiaries of a social security scheme in order to be included.

Non-inclusion criteria

Exclusion criteria include refusal to participate, inability to follow-up at day 14 and any conditions making lung 
Panel A.
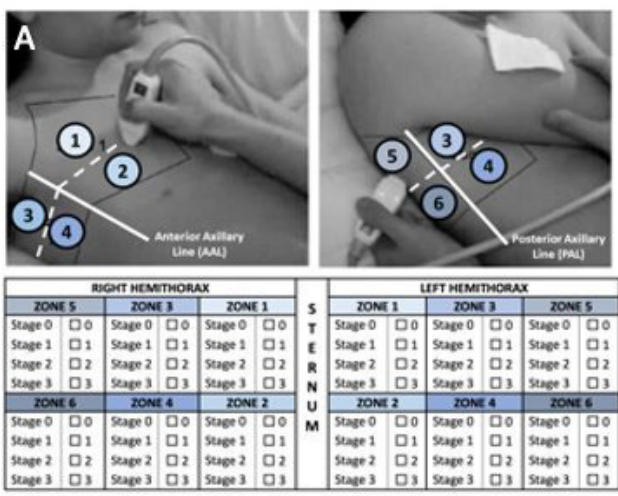

B

L-POCUS score
Panel B.
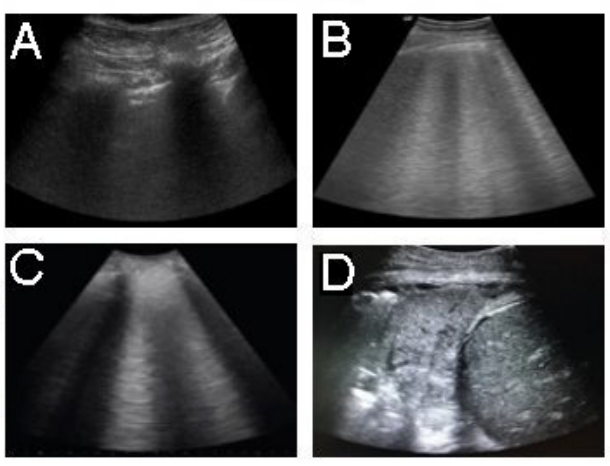

Figure 1 Panel A: lung point-of-care ultrasonography method (L-POCUS): (A) Twelve chest areas of investigation following BLUE-PLUS protocol: zone 1: upper anterior chest wall; zone 2: lower anterior chest wall; zone 3: upper lateral chest wall; zone 4: lower lateral chest wall; zone 5: upper posterolateral chest wall; zone 6: lower posterolateral chest wall. (B) L-POCUS score grid: four ultrasound parenchymal aeration stages are searched in each zone, and points are affected to them according to their severity. Stage 0 or normal aeration (0 point): line sliding sign associated with respiratory movement or less than three B lines; stage 1 or moderate loss of lung aeration (1 point): a clear number of multiple visible B lines with horizontal spacing between adjacent $B$ lines $\leq 7 \mathrm{~mm}$ (B1 lines); stage 2 or severe loss of lung aeration (2 points): multiple B lines fused together that were difficult to count with horizontal spacing between adjacent B lines $\leq 3 \mathrm{~mm}$, including 'white lung'; and stage 3 or pulmonary consolidation (3 points): hyperechoic lung tissue, accompanied by dynamic air bronchogram. Panel B: examples of four ultrasound aeration stages. (A) Stage 0 or normal aeration; (B) stage 1 or moderate loss of lung aeration; (C) stage 2 or severe loss of lung aeration; and (D) stage 3 or pulmonary consolidation.

ultrasonography impossible (body mass index $>35 \mathrm{~kg} / \mathrm{m}^{2}$, history of pneumonectomy and so on).

\section{Lung point-of-care ultrasonography}

Lung POCUS is performed by trained practitioners with ultrasound scanners using the following parameters: low frequency $(2-5 \mathrm{MHz})$ transductors, convex (abdominal transductors) or small linear (cardiac transductors) type probes that optimally explore at the thoracic depth from $6 \mathrm{~cm}$ to $10 \mathrm{~cm}$. This includes patient L-POCUS performed within 48 hours following admission to the emergency department. Considering the COVID-19 pandemic, special protective precautions are respected to limit the risk of contamination between the patient and the operator (disposable single-use personal protective equipment, single-use ultrasound probe protection covers, cleaning and antiviral disinfection before and after each use). L-POCUS is performed using the BLUEPLUS Protocol 12 regions method ${ }^{15}$ investigated in a semirecumbent or supine position (figure 1, panel A). All intercostal spaces of the upper and lower parts of the anterior, lateral and posterior regions of the left and right chest walls were examined, resulting in 12 areas of investigation. Four ultrasound aeration stages are defined ${ }^{1421}$ (figure 1, panel B): stage 0 or normal aeration: line sliding sign associated with respiratory movement or less than three B lines; stage 1 or moderate loss of lung aeration: a clear number of multiple visible B lines with horizontal spacing between adjacent $\mathrm{B}$ lines $\leq 7 \mathrm{~mm}$ (B1 lines); stage 2 or severe loss of lung aeration: multiple B lines fused together that were difficult to count with horizontal spacing between adjacent B lines $\leq 3 \mathrm{~mm}$, including 'white lung'; and stage 3 or pulmonary consolidation: hyperechoic lung tissue, accompanied by dynamic air bronchogram. The L-POCUS score is determined by allocating $0,1,2$ or 3 points to parenchymal aeration stages $0,1,2$ or 3 respectively in every area. Each of the 12 lung areas is examined, and the final L-POCUS aeration score, ranging from 0 to 36 points, is the sum of each regional ultrasound score. We also determine the presence of pleural effusion, or absence thereof, for each hemithorax.

\section{Trial outcomes}

\section{Primary outcome}

The primary outcome is the occurrence of death, regardless of cause, or the use of intubation or invasive ventilation within the 14 days (day 14) following inclusion. The ability of POCUS to detect the primary outcome occurrence will be evaluated by determining the $95 \% \mathrm{CI}$ of the area under the curve (AUC) of the receiver operating characteristic (ROC) curve. L-POCUS prognostic value will be considered as clinically relevant if the lower bound of the $95 \% \mathrm{CI}$ is equal or greater than 0.7 .

A sensitivity analysis is performed with the 14-day allcause mortality rate as outcome.

\section{Secondary outcomes}

The secondary outcomes include the following parameters:

- The risk of unfavourable outcome (occurrence of death or the use of intubation or invasive ventilation) over time - that is, the L-POCUS performances according to the delay of outcome assessment. This involves evaluating, in patients with a confirmed or probable SARS-CoV-2 infection, whether L-POCUS score performances vary depending on time, between day 1 and day 14 and, if so, until which time horizon its performances are clinically 
relevant. For this purpose, we will determine the period for which the lower limit of the $95 \%$ CI of the AUC of the L-POCUS score ROC curve is at least 0.7.

- The risk stratification threshold values of L-POCUS score defining three risk groups: low-risk patients, intermediaterisk patients and high-risk patients. For this purpose, we will determine two threshold values: first, maximising the specificity for a sensitivity of at least $95 \%$, and second, maximising the sensitivity for a specificity of at least $95 \%$.

- Effect of adding L-POCUS score value to previous several risk stratification clinical rules for pulmonary infection or sepsis: qSOFA, CRB 65 and CURB 65. For this purpose, we will attribute 0,1 or 2 points to L-POCUS score according to the two predefined threshold values and will assess the AUCs of qSOFA, CRB 65 and CURB 65 with and without addition of L-POCUS score result.

- L-POCUS score evolution performances in the subgroup of hospitalised patients having a second chest ultrasonography at day $5 \pm 3$ days of inclusion. We will assess the performances of the L-POCUS score evolution between the first and the second assessment in identifying patients with unfavourable outcome (intubation and mechanical ventilation requirement or death). For this purpose, we will calculate the delta between the first and second L-POCUS score and determine the AUC of the ROC curve and its $95 \%$ CI.

- L-POCUS score performances ability to predict the risk of unfavourable outcome in the subgroup of patients with positive SARS-CoV-2 RT-PCR results.

\section{Participant timeline}

Study participation duration for a participant is 14 days.

\section{Sample size}

To study diagnostic performances of lung ultrasound to identify high-risk patients, we will determine the $95 \%$ CI of the AUC of the ROC curve and consider L-POCUS capacity as clinically relevant if the lower limit of the $95 \%$ CI is at least 0.7. This is assuming that the observed AUC will be 0.8 . Based on data from COVID-19 in China, the rate of death or need for tracheal intubation is estimated at 20\% in high-risk patients. As severely ill critical patients are excluded of our study, we estimate that this rate will be around $10 \%$. Therefore, assuming a rate of death or tracheal intubation requirement of $10 \%$, and expecting an AUC of 0.8, the number of patients required to achieve a lower limit of the $95 \%$ CI upper than 0.7 was estimated to 286. Taking into consideration patients lost to follow-up and those who cannot be evaluated (estimated at 5\%), it is necessary to include 300 patients in total.

\section{Recruitment}

Inclusions started on 10 April 2020 in Angers University Hospital and 11 centres had included at least one patient by 18 April 2020. Taking into account the number of participating centres (11) and the evolution of COVID-19, the estimated duration of inclusion is 3 months.

\section{Patient and public involvement}

This research was done without patient involvement. Patients were not invited to comment on the study design and were not consulted to develop patient relevant outcomes or interpret the results. Patients were not invited to contribute to the writing or editing of this document for readability or accuracy.

\section{Data collection, management and analysis}

Data collection and management

All data related to this study are collected using a standardised electronic case report form (eCRF) and based on valid documents (patient medical record). In the eCRF and follow-up calls, patients can be identified by a unique number composed of the centre number and the patient number at the centre. The confidentiality of patients and their personal health information is always maintained by restricting access to patient records and eCRF.

\section{Statistical analysis}

Many different data sets are described in this study. Quantitative data are described using means and SD. Qualitative data are described using numbers, percentages and 95\% CIs. L-POCUS properties to predict unfavourable outcome over time is estimated by calculating the AUC and its $95 \%$ CI. Sensitivity and specificity will be estimated by the $632+$ bootstrap method. Calibration will be assessed with the calibration slope and the calibration intercept. A flexible calibration curve will be provided. A Brier score will be also reported, summarising the magnitude of error in the probability forecasts between 0.0 and 1.0 , where a perfectly calibrated model would score 0.0. Two thresholds will be calculated. The first will maximise specificity with a sensitivity greater than or equal to $95 \%$, and the second will maximise sensitivity with a specificity greater than or equal to $95 \%$. For these threshold values, we will present sensitivity, specificity, predictive values and likelihood ratios. Dynamic changes of L-POCUS diagnostic properties will be realised depending on the time AUC and also its $95 \%$ CI. To study the impact of adding the result of L-POCUS evaluation to several risk stratification clinical rules for pulmonary infection or sepsis (qSOFA, CRB 65 and CURB 65), AUCs will be compared with or without its component with a DeLong test. In this purpose, we will attribute 0,1 or 2 points to L-POCUS result as low, moderate or high risk according to the predefined thresholds values and assessed the AUC of the risk stratification rules with and without adding the L-POCUS result value. Calibration of these rules will also be assessed with the calibration slope and calibration intercept.

\section{ETHICS AND DISSEMINATION Legal obligations and approval}

The sponsor of the study is CHU d'Angers (Angers University Hospital). The sponsor obtained prior approval from the Comité de Protection des Personnes (CPP) du Sud-Ouest et Outre-Mer 2 (n ID-RCB: 2020-A00782-37/2-20-025 id7566, 3 April 2020) and the Belgian Comite d'Ethique Hospitalo-Facultaire des Cliniques universitaires Saint-Luc (2020/14AVR/223, 15 April 2020). The Declaration of 
Helsinki and the Good Clinical Practice guidelines will be respected by the study. The coordinating investigator can make an amendment after submission to the sponsor and approval from the CPP. After complying with these different stages, the amendment will be implemented.

\section{Dissemination of results}

Considering the ongoing COVID-19 pandemic, the main result of the study regarding the lung ultrasound performances to predict unfavourable development will be deposited on a preprint server and presented in a peer-reviewed journal as soon as possible. This study adhere to TRIPOD guidelines, by verifying the 22 items of TRIPOD checklist during the conception of this protocol. ${ }^{22}$ The Strengthening the Reporting of Observational Studies in Epidemiology statement will be used before submitting the manuscript to a journal. $^{23}$

The full results of the study will be presented in national and international meetings and in peerreviewed journals.

\section{Trial status}

Inclusions started on 10 April 2020.

\section{DISCUSSION}

This study protocol describes a prospective and multicentre study evaluating POCUS for risk stratification of patients with COVID-19.

While the usefulness of ultrasound for standard organ examinations has been shown and unanimously accepted for a long time, the lung ultrasound has traditionally been excluded from this repertoire. ${ }^{24}$ Ultrasound techniques have expanded, and their usefulness has been gradually demonstrated and democratised worldwide by the works of Lichtenstein and Mezière ${ }^{1521}$ and Volpicelli et al. ${ }^{25} 26$

Many articles have been published on this topic in the context of the COVID-19 pandemic but mostly based on expert opinion without evidence based data. ${ }^{27-30}$ Buonsenso et al even suggest that lung ultrasounds could replace stethoscopes in the ongoing COVID-19 pandemic, which could possibly reduce the risk of exposure of healthcare workers. ${ }^{31}$ Its main advantages for COVID-19 can be seen ${ }^{28}$ in the following stages: (1) triage (pneumonia/non-pneumonia) of symptomatic patients at home as well as in the prehospital phase; (2) diagnostic suspicion and awareness in the emergency department setting; and (3) treatment of intensive care unit patients with regard to ventilation and weaning. Therefore, as highlighted by Soldati $e t a l^{28}$ studies aimed at clarifying the diagnostic and prognostic role of lung POCUS in COVID-19 are urgently needed. This is the principal aim of our study.

Lung ultrasound will also likely make it possible to monitor the clinical course of COVID-19 patients and the effects of therapeutic measures. ${ }^{32}$ This is the reason why we designed our study by integrating a monitoring ultrasound at day $5 \pm 3$.

The potential advantages of L-POCUS are important, especially versus lung CT scan. However, the scientific community warns us about its limitations and pitfalls, ${ }^{33} 34$ meaning that we need an adequately designed study to determine the limits and advantages of this tool. POCUSCO may likely provide part of the expected answers.

There are some limitations in the conception of this study. First, at the time we wrote the protocol and on the basis of the first observational studies in China, the expected rate of mortality or intubation and invasive ventilation request was set to $10 \% .^{18}$ However, recent data have demonstrated mortality rates lower than those observed in the early phases of the epidemic in Wuhan. ${ }^{135}$ Moreover, the efficacy of some treatments as corticosteroids has been recently proved in COVID- $19 .^{36}$ As a result, the rate of our primary outcome may be lower than expected, impairing the powerful of our trial and enlarging the 95\% CIs of our estimates. Second, as only patients with a mild to moderate COVID-19 will be included, most of them may be discharged home without the possibility to perform a second L-POCUS at day $5 \pm 3$ following the inclusion. Therefore, the assessment of the performances of the L-POCUS score evolution may be limited. A final limitation is related to POCUS itself, all operators not having the same level of experience in POCUS realisation and all hospitals not using the same devices. ${ }^{37}$ The interindividual variability in the L-POCUS score grading may then be increased. The L-POCUS performances may be underevaluated as compared within an expert centre, but our results will show the performances that should be expected in real life care

The POCUSCO study results are particularly anticipated, and after the protocol was reviewed and approved by French and Belgian Ethics Committee, recruitment began on 10 April 2020. The results are anticipated for the end of June 2020.

\section{Author affiliations}

${ }^{1}$ Department of Emergency Medicine, University Hospital of Angers, Univ Angers, Angers, France

${ }^{2}$ Institut MitoVasc, UMR CNRS 6215 INSERM 1083, Angers, France

${ }^{3}$ Department of Methodology and Biostatistics, University Hospital of Angers, Univ Angers, Angers, France

${ }^{4}$ Department of Pediatric Oncology, University Hospital of Angers, Univ Angers, Angers, France

${ }^{5}$ Department of Emergency Medicine, Cliniques Universitaires Saint-Luc, Brussels, Belgium

${ }^{6}$ IRSET (Institut de recherche en santé, environnement et travail) - UMR_S 1085, F-49000, Angers, France

${ }^{7}$ Department of Radiology, University Hospital of Angers, Univ Angers, Angers, France

${ }^{8}$ Infectious Diseases and Tropical Medicine, University Hospital of Angers, Univ Angers, Angers, France

Twitter François Morin @Francois_Mo_49 
Acknowledgements We would like to thank all the team of the 'Maison de la Recherche Clinique' of CHU d'Angers and especially Sandra Merzeau and JeanMarie Chrétien. We would also like to thank all the research team of the Emergency Department of Angers University Hospital, and especially Cindy Augereau, Chloé Ragueneau, Clothilde Aubert and Barbara Maquin.

Contributors FM, PM-R, VD, J-FH and DD conceived and designed the study. All authors participated in the design of POCUSCO and contributed to revisions of the original manuscript. J-FH, FM and JeR performed the statistical plan and sample size calculation. FM, DD, FD, DS, VD and P-MR realised acquisition of data. $\mathrm{J}-\mathrm{FH}$ and FM performed data analysis and interpretation. All authors edited the manuscript and read and approved the final manuscript.

Funding The trial is funded by a grant from the French Ministry of Health (PHRC-I 2020,COVID19_A_001, 26 May 2020).

Disclaimer The funding source had no role in the design of the trial and will have no role in its execution, in the analysis and interpretation of data, or in the decision to submit results for publication.

\section{Competing interests None declared.}

Patient and public involvement Patients and/or the public were not involved in the design, or conduct, or reporting, or dissemination plans of this research.

\section{Patient consent for publication Obtained.}

Provenance and peer review Not commissioned; externally peer reviewed.

Open access This is an open access article distributed in accordance with the Creative Commons Attribution Non Commercial (CC BY-NC 4.0) license, which permits others to distribute, remix, adapt, build upon this work non-commercially, and license their derivative works on different terms, provided the original work is properly cited, appropriate credit is given, any changes made indicated, and the use is non-commercial. See: http://creativecommons.org/licenses/by-nc/4.0/.

\section{ORCID iDs}

François Morin http://orcid.org/0000-0002-1582-2934

Vincent Dubée http://orcid.org/0000-0002-9982-4741

Pierre-Marie Roy http://orcid.org/0000-0003-4811-6793

\section{REFERENCES}

1 Epidemiology Working Group for NCIP Epidemic Response, Chinese Center for Disease Control and Prevention. [The epidemiological characteristics of an outbreak of 2019 novel coronavirus diseases (COVID-19) in China]. Zhonghua Liu Xing Bing Xue Za Zhi 2020;41:145-51.

2 Wu Z, McGoogan JM. Characteristics of and important lessons from the coronavirus disease 2019 (COVID-19) outbreak in China: summary of a report of 72314 cases from the Chinese center for disease control and prevention. JAMA.

3 Guan W-J, Z-Y N, Hu Y. Clinical characteristics of coronavirus disease 2019 in China. N Engl J Med.

4 Rodriguez-Morales AJ, Cardona-Ospina JA, Gutiérrez-Ocampo E. Clinical, laboratory and imaging features of COVID-19: a systematic review and meta-analysis. Travel Med Infect Dis 2020;101623.

5 Ding X, Xu J, Zhou J, et al. Chest CT findings of COVID-19 pneumonia by duration of symptoms. Eur J Radiol 2020;127:109009.

6 Bernheim A, Mei X, Huang M, et al. Chest CT findings in coronavirus Disease-19 (COVID-19): relationship to duration of infection. Radiology 2020;295:200463.

$7 \mathrm{Ai} \mathrm{T}$, Yang Z, Hou H, et al. Correlation of chest CT and RT-PCR testing for coronavirus disease 2019 (COVID-19) in China: a report of 1014 cases. Radiology 2020;296:E32-40.

8 Li K, Fang Y, Li W. Ct image visual quantitative evaluation and clinical classification of coronavirus disease (COVID-19). Eur Radiol.

9 Yuan M, Yin W, Tao Z, et al. Association of radiologic findings with mortality of patients infected with 2019 novel coronavirus in Wuhan, China. PLoS One 2020;15:e0230548.

10 Haute Autorité de la Santé, Société d'Imagerie Thoracique (SIT), collège des Enseignants en Radiologie de France (CERF). Indications Du scanner thoracique: réponses rapides dans Le cadre Du COVID-19 2020.

11 Dennie C, Hague C, Lim RS, et al. Canadian Society of thoracic Radiology/Canadian association of radiologists consensus statement regarding chest imaging in suspected and confirmed COVID-19. Can Assoc Radiol J 2020;71:470-81.
12 National Health Commission of the People's Republic of China. Diagnosis and treatment of novel coronavirus pneumonia, 2020. Available: http://www.nhc.gov.cn/yzygj/s7653p/202002/3b09b894 ac9b4204a79db5b8912d4440.shtml

13 Schenck EJ, Rajwani K. Ultrasound in the diagnosis and management of pneumonia. Curr Opin Infect Dis 2016;29:223-8.

14 Zhao Z, Jiang L, Xi X, et al. Prognostic value of extravascular lung water assessed with lung ultrasound score by chest sonography in patients with acute respiratory distress syndrome. BMC Pulm Med 2015;15:98.

15 Lichtenstein DA, Mezière GA. Relevance of lung ultrasound in the diagnosis of acute respiratory failure: the BLUE protocol. Chest 2008;134:117-25.

16 Mohamed MFH, Al-Shokri S, Yousaf Z, et al. Frequency of abnormalities detected by point-of-care lung ultrasound in symptomatic COVID-19 patients: systematic review and metaanalysis. Am J Trop Med Hyg 2020;103:815-21.

17 Nouvenne A, Zani MD, Milanese G, et al. Lung ultrasound in COVID-19 pneumonia: correlations with chest CT on hospital admission. Respiration 2020;99:617-24.

18 Zhou F, Yu T, Du R, et al. Clinical course and risk factors for mortality of adult inpatients with COVID-19 in Wuhan, China: a retrospective cohort study. Lancet 2020;395:1054-62.

19 World Health Organisation. Clinical management of severe acute respiratory infection (SARI) when COVID-19 disease is suspected, 2020. Available: https://www.who.int/publications-detail-redirect/ clinical-management-of-covid-19 [Accessed 6 Jul 2020].

20 World Health Organisation. WHO. R\&D blueprint - novel Coronavirus - COVID-19 therapeutic trial synopsis, 2020. Available: https://www.who.int/blueprint/priority-diseases/key-action/COVID19_Treatment_Trial_Design_Master_Protocol_synopsis_Final_ 18022020.pdf

21 Lichtenstein D. Échographie Pulmonaire en réanimation et aux urgences. Réanimation 2008;17:722-30.

22 Moons KGM, Altman DG, Reitsma JB, et al. Transparent reporting of a multivariable prediction model for individual prognosis or diagnosis (TRIPOD): explanation and elaboration. Ann Intern Med 2015;162:W1-73.

23 Cuschieri S. The STROBE guidelines. Saudi J Anaesth 2019;13:31-4

24 Fuhlbrigge AL, Choi AMK. Diagnostic procedures in respiratory disease. In: Kasper D, Fauci A, Hauser S, et al., eds. Harrison's principles of internal medicine. New York: McGraw-Hill Education, 2014. accessmedicine.mhmedical.com/content.aspx?aid= 1120807489

25 Volpicelli G, Caramello V, Cardinale L, et al. Bedside ultrasound of the lung for the monitoring of acute decompensated heart failure. Am J Emerg Med 2008;26:585-91.

26 Volpicelli G, Elbarbary M, Blaivas M, et al. International evidencebased recommendations for point-of-care lung ultrasound. Intensive Care Med 2012;38:577-91.

27 Moore S, Gardiner E. Point of care and intensive care lung ultrasound: a reference guide for practitioners during COVID-19. Radiography 2020;26:e297-302.

28 Soldati G, Smargiassi A, Inchingolo R. Is there a role for lung ultrasound during the COVID-19 pandemic? J Ultrasound Med.

29 Lomoro P, Verde F, Zerboni F, et al. COVID-19 pneumonia manifestations at the admission on chest ultrasound, radiographs, and CT: single-center study and comprehensive radiologic literature review. Eur J Radiol Open 2020;7:100231.

30 Sofia S, Boccatonda A, Montanari M. Thoracic ultrasound and SARS-COVID-19: a pictorial essay. J Ultrasound.

31 Buonsenso D, Pata D, Chiaretti A. COVID-19 outbreak: less stethoscope, more ultrasound. Lancet Respir Med 2020;8:e27.

32 Kulkarni S, Down B, Jha S. Point-of-care (POC) lung ultrasound in intensive care during the COVID-19 pandemic. Clin Radiol 2020;75:710.e1

33 Cheung JC-H, Lam KN. POCUS in COVID-19: pearls and pitfalls. Lancet Respir Med 2020;8:e34.

34 khalili N, Haseli S, Iranpour P. Lung ultrasound in COVID-19 pneumonia; prospects and limitations. Acad Radiol.

35 Cao B, Wang Y, Wen D, et al. A trial of lopinavir-ritonavir in adults hospitalized with severe Covid-19. N Engl J Med 2020;382:1787-99.

36 Sterne JAC, Murthy S, et al, WHO Rapid Evidence Appraisal for COVID-19 Therapies (REACT) Working Group. Association between administration of systemic corticosteroids and mortality among critically ill patients with COVID-19: a meta-analysis. JAMA.

37 Rouby J-J, Arbelot C, Gao Y, et al. Training for lung ultrasound score measurement in critically ill patients. Am J Respir Crit Care Med 2018;198:398-401. 